\title{
Adhesion and proliferation of skeletal muscle cells on single layer poly(lactic acid) ultra-thin films
}

\author{
Leonardo Ricotti • Silvia Taccola • Virginia Pensabene • \\ Virgilio Mattoli • Toshinori Fujie • Shinji Takeoka • \\ Arianna Menciassi • Paolo Dario
}

(C) Springer Science+Business Media, LLC 2010 muscle tissue engineering, drug delivery, and - in generalin the field of bio-hybrid devices.

Keywords Nanofilms · Biocompatibility · Poly(lactic acid) $\cdot$ Skeletal muscle cells $\cdot \mathrm{C} 2 \mathrm{C} 12 \cdot$ Bio-hybrid devices

\section{Introduction}

In the last 10 years microorganisms, muscle cells and cardiomyocytes have been utilized as drivers for cell-based actuators, which consist of a rigid or flexible artificial substrate in the micrometer scale actuated by the natural energy and motion of the cells. A typical feature of all cellbased bio-microactuators is that they are self-actuated, wireless mechanochemical transducers requiring no externally coupled energy sources, unlike conventional actuators. A challenging feature is the complete integration of a cell-based actuator, as its energy source is represented by the chemical energy of the nutrients, "embedded" in the cellular aqueous environment. Cells and micromechanical systems can be combined to develop microdevices (Tanaka et al. 2007a (I)), such as transport systems, rotating or oscillating structures, swimming or walking robots and microfluidic pumps (Weibel et al. 2005; Hiratsuka et al. 2006; Herr and Dennis 2004; Xi et al. 2005; Tanaka et al. 2006). Consequently, artificial substrates for bio-actuated systems find application not only in tissue engineering, but also as novel substrate concepts to enhance microactuator performances and usability.

Most systems in literature use cardiomyocytes or cardiomyocytes sheets as actuating element, because these cells contract spontaneously, thus representing intrinsically pulsatile microactuators. The regular and robust pulsatile phenotype of these cells allows the realization of interesting 
devices (Morishima et al. 2006; Tanaka et al. 2007b (II); Kim et al. 2006). The controllability of the system, in terms of frequency, amplitude and period of the stimuli, can be achieved using not-spontaneous contractile cell lines, such as $\mathrm{C} 2 \mathrm{C} 12$ murine myoblasts (Blau et al. 1983), which is a common model for muscle biology and regeneration studies (Bach et al. 2004). Both in vitro and in vivo, the differentiation process and the following new muscle formation require that mononucleated muscle precursor cells proliferate onto the substrate, fuse together into multinucleated syncytia, and finally differentiate into elongated structures, called myotubes.

After their differentiation in myotubes, it is possible to stimulate the contractile activity of $\mathrm{C} 2 \mathrm{C} 12$ cells by using external electrical pulses, as described by Fujita (Fujita et al. 2007) on a porous membrane-based cell culture device. Typically, the electrical stimulation of cultured muscle cells on a dish is performed using a pair of stimulating electrodes placed at both side of the chamber (Sathaye et al. 2006; Wilson et al. 2007; Vandenburgh et al. 2008), but by using rigid substrates the muscle contraction is obviously isometric, as the substrate cannot deform under the relatively small force generated by the cells. The orientation of the stimulating electrical field respect to the myotubes axis plays also an important role (Ishibashi et al. 2008) and, in general, the contraction depends on the pulse frequency and reflects the anisotropy of the electric field. Yamasaki quantitatively investigated the effect of electrical pulse frequency on the excitability of myotubes (Yamasaki et al. 2009). The obtained results lead towards the realization of controllable muscle-actuated devices based on $\mathrm{C} 2 \mathrm{C} 12$ skeletal muscle cells.

Coupling cells with a flexible substrate is a complex issue, regulated by a precise balance between the specific cell behavior and the chemical, physical and mechanical properties of the substrate. The first event occurring when a cell contacts a material is adhesion. Several works highlighted the possibility to tune this phase by modifying the substrate, adding chemical and physical cues which replicate the natural features of the extracellular matrix (Hubbell 1999). Concerning the surface morphology, it has been demonstrated that cells adhesion is very sensitive to variations in nanometer-scale topography (Raffa et al. 2007). An example is given by Washburn (Washburn et al. 2004) indicating that nanoroughness values ranging from 0.5 to $13 \mathrm{~nm}$ influence the adhesion of MC3T3-E1 osteoblastic cells on substrates coated with poly(L-lactic acid) (PLLA) films. In addition, controlling the surface properties of the material is of crucial importance, as they can also influence proliferation, differentiation and spreading (Shin et al. 2003). In particular, within a tissue or in in vitro cultures, cells grow not only by applying forces, but also responding through cytoskeleton organization to the resistance deriving from normal tissue matrix, synthetic substrate, or even adjacent cells (Discher et al. 2005). This is particularly true for contractile cells, such as differentiated muscle cells, whose function is also related to the stiffness of the matrix. Myotubes ultimately transmit actomyosin contractions through their attachments to the matrix, and it has been demonstrated that initial myoblast adhesion, proliferation, and subsequent myoblast differentiation of skeletal muscle cells into myotubes depend intimately on optimal outside-in signaling of matrix elasticity (Engler et al. 2004).

The contraction of tissue cells was already observed on free-standing micrometric polydimethylsyloxane (PDMS) flexible films (Feinberg et al. 2007), due to the transmission of the traction forces exerted by the cell phenotype to the matrix, occurring primarily through specialized adhesion and cytoskeletal linkages. The cellular forces produce substrate displacement, but only if allowed by the appropriate mechanical properties of the substrate. Furthermore the role of thin film mechanical properties (with film thickness in the micrometric range) on cell differentiation has been elucidated using multilayer films made of poly $(\mathrm{L}$ lisine)/Hyaluronan (PLL/HA) over a large range of film stiffness (Ren et al. 2008). However, the use of freestanding growth scaffold with nanometric thickness has still to be investigated, as their use as flexible supports for cellbased actuators.

Polymeric single and multilayer ultra-thin films (also called "nanofilms") were recently developed and soon after they drew the attention of many research groups (Tang et al. 2006). They can be prepared by spin coated assisted deposition, first delivering a sacrificial layer which can be dissolved in water or in a specific solvent, thus enabling the detachment of the film from the substrate and the manipulation of the nanofilm in liquid. By spin coating deposition, plenty of polymers can be used, by obtaining biocompatible and resistant sheets (Fujie et al. 2007). Thanks to the controllability of the thickness, which is related to the production components, to the kind of polymer and it is also linearly dependent on the number of layers, nanofilms show ultra-thin thickness, large area, and elastic modules up to the GPa (Vendamme et al. 2006).

We investigate the behavior of a single layer ultra-thin film as substrate for cell-based devices, in order to identify a scaffold able to easily follow the contraction and the relative movement induced by the cells cultured and differentiated on its surface. The study of adhesion and proliferation is obviously a prerequisite to cell differentiation, and we approach these specific aspects in this work. We first characterized single layer PLA nanofilms, in terms of thickness and roughness affecting the mechanical and structural properties. We also evaluated the elastic modulus 
of the nanofilms. We report our results as regards $\mathrm{C} 2 \mathrm{C} 12$ cells cultured on the nanofilms, without any surface coating or functionalization, thus evaluating short and medium term biocompatibility, cell adhesion, proliferation and observing early differentiation into myotubes.

\section{Materials and methods}

\subsection{Fabrication and characterization of PLA nanofilms}

Free-standing polymeric nanofilms were synthesized by spin coated assisted deposition following the procedure described in (Pensabene et al. 2009). Briefly silicon wafers $\left(\mathrm{SiO}_{2}\right.$ substrates, from Si-Mat Silicon Materials, Landsberg AM Lech, Germany), were cut $\left(2 \mathrm{~cm}^{2}\right)$ and cleaned for 10 min with a mixture of sulfuric acid and hydrogen peroxide (3:1) and then thoroughly rinsed by deionised water. All preparation routines for polymeric nanofilms fabrication were conducted in a clean-room (class 1000) to avoid contamination. A poly(vinyl alcohol) aqueous solution (PVA 1 wt.\%, average Mw 13,000-23,000, 98\% hydrolyzed, Sigma-Aldrich) was deposed by spin-coating on a silicon wafer (at 4,000 rpm for $20 \mathrm{~s}$ ) forming the sacrificial layer of water-soluble polymer. A following solution of poly (lactic acid) in dichloromethane (PLA 2 wt.\%, Mw $\sim 60,000$, Sigma-Aldrich) was then spin-coated on the first layer by using the same spinning parameters. After drying the sample (at $80^{\circ} \mathrm{C}$ for $1 \mathrm{~min}$ ), the PVA sacrificial layer was dissolved in water thus releasing the PLA nanofilm free-standing in liquid (Fig. 1(a)).

For measuring the film thickness and the surface roughness by Atomic Force Microscopy (AFM), the freesuspended films were collected and dried onto a fresh silicon wafer (Fig. 1(b)). Thickness values were obtained by AFM cross-sectional analysis of nanofilm edge (SPMLab software Version 5.01). Imaging was performed with Veeco Innova Scanning Probe Microscope operating in tapping mode, with oxide-sharpened silicon probes (RTESPA-CP) at resonant frequency of $\approx 300 \mathrm{kHz}$. The mechanical properties of the single layer nanofilms were evaluated by a bulging test, described in details in the literature (Huang et al. 2007). The experimental setup for the measurement of the elastic modulus is shown in Fig. 1bis. A free-standing nanofilm floating in water was deposed and dried on a steel plate with a circular hole in the middle, and air pressure was applied to the nanomembrane from backside through the hole. Applied overpressure was monitored by a digital manometer, and the deflection behavior of the nanofilm was observed with an optical microscope, until distortion was apparent. Microscopic pictures in Fig. 2(b)-(d) are examples of the deflected nanofilm.

The value of the stress $(\sigma)$, the strain $(\varepsilon)$ and the elastic modulus (E) of the nanofilm were determinated using the following equations

$$
\begin{aligned}
& \sigma=\frac{P a^{2}}{4 h d} \\
& \varepsilon=\frac{2 d^{2}}{3 a^{2}} \\
& E=\frac{\sigma}{\varepsilon}
\end{aligned}
$$

where $\mathrm{P}$ is the pressure applied, $\mathrm{a}$ is the radius of the metal hole $(3 \mathrm{~mm}), \mathrm{h}$ is the nanofilm thickness and $\mathrm{d}$ is the deflection of the nanofilm. From the initial elasticity of the stress-strain curve (Fig. 2(e)), the elastic modulus was calculated.

\section{$2.2 \mathrm{C} 2 \mathrm{C} 12$ cells seeding and nanofilm preparation}

C2C12 mouse myoblasts (ATCC, CRL-1772) were cultured in Dulbecco's modified Eagle's medium (DMEM, ATCC) supplemented with $10 \%$ fetal bovine serum (FBS, ATCC), $100 \mathrm{IU} / \mathrm{ml}$ penicillin, $100 \mu \mathrm{g} / \mathrm{ml}$ streptomycin and $2 \mathrm{mM} \mathrm{L-}$ glutamine. Cells were maintained at $37^{\circ} \mathrm{C}$ in a saturated humidity atmosphere containing 95\% air / 5\% $\mathrm{CO}_{2}$, and they were subcultured before reaching $60-70 \%$ confluence
Fig. 1 PLA nanofilm: (a) released and floating on water surface; (b) lifted-up by a silicon substrate $($ scale bar $=1 \mathrm{~cm}$ )
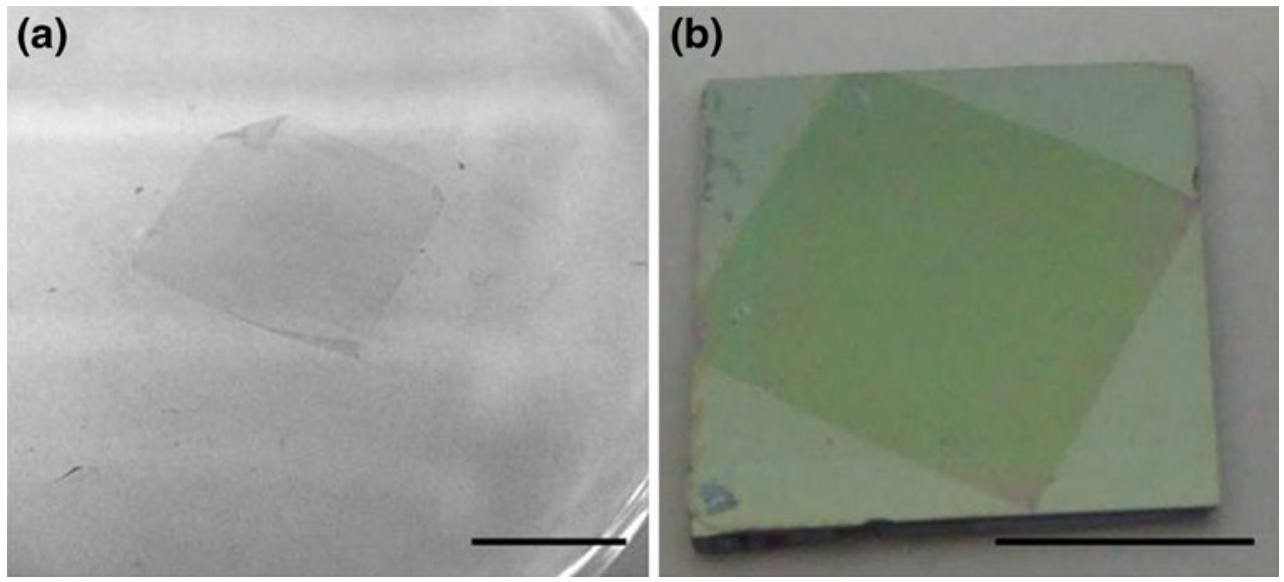


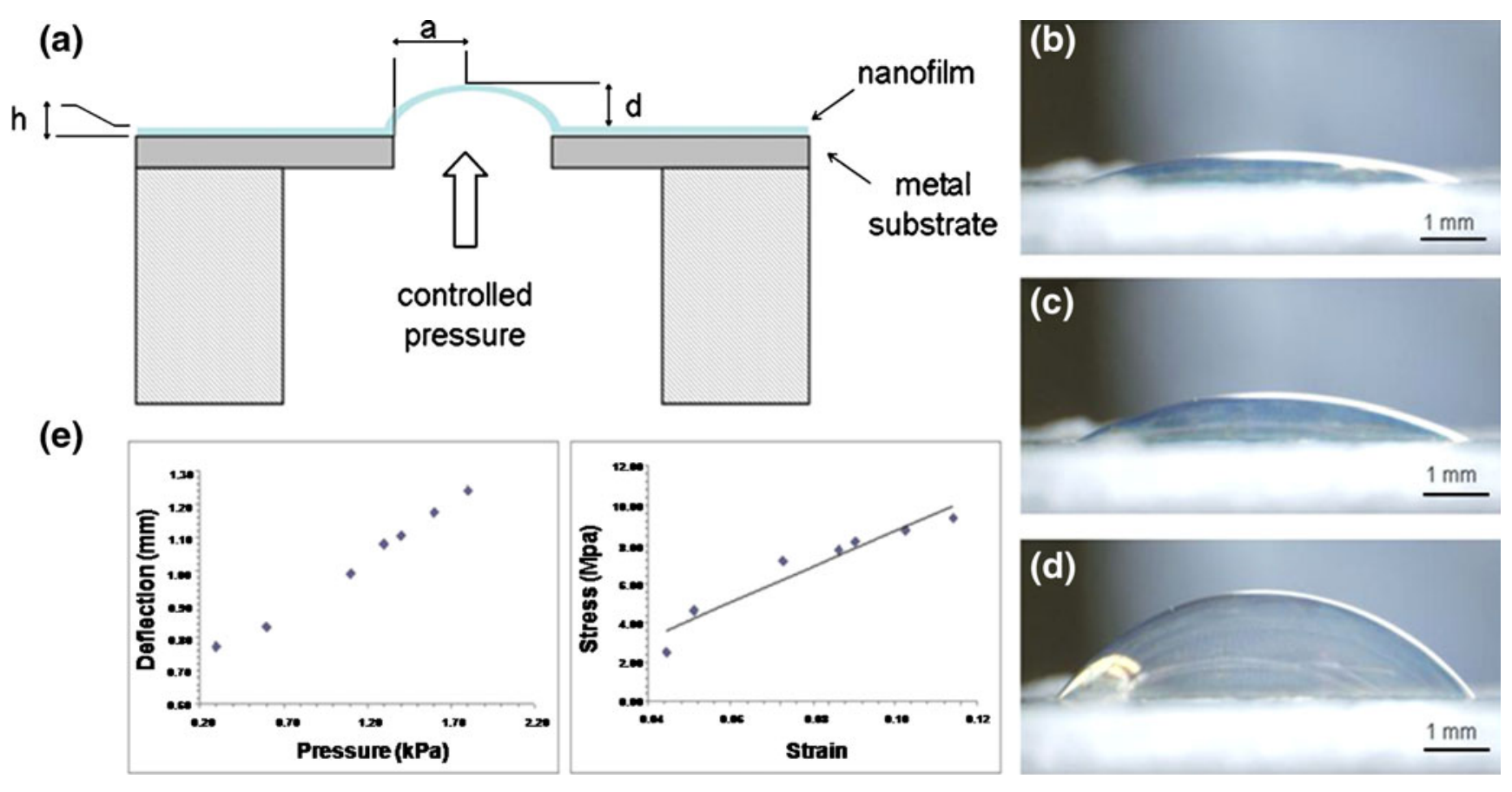

Fig. 2 Bulging experiments; (a) experimental setup; (b)-(d) Sequential macroscopic cross-sectional views of the deflected nanofilm when different pressures were applied through the $6 \mathrm{~mm}$ diameter hole; (e) corresponding pressure-deflection and stress-strain curves

(approximately every 2 days). Differentiation into myotubes was induced by switching $80-90 \%$ confluent cells to DMEM supplemented with $1 \%$ FBS, $1 \%$ ITS liquid media supplement (Sigma-Aldrich, I3146), consisting of a mixture of bovine insulin, human transferrin, sodium selenite and ethanolamine, $100 \mathrm{IU} / \mathrm{ml}$ penicillin, $100 \mu \mathrm{g} / \mathrm{ml}$ streptomycin and $2 \mathrm{mM}$ L-glutamine (Yaffe and Saxel 1977). Before C2C12 seeding, nanofilms were immersed in de-ionized water and detached from the Si wafer, in a sterile Petri dish. Sterilization of the floating nanofilms was performed by means of UV rays for $45 \mathrm{~min}$. Deionized water was then replaced with culture medium, maintaining the nanofilms floating on the liquid surface. Cells $\left(160,000 / \mathrm{cm}^{2}\right)$ were seeded on the surface of each nanofilm, and after $12 \mathrm{~h}$ the nanofilms with attached cells were moved in fresh culture medium, in order to discard the cells on the original Petri dish bottom and to observe the behavior of the cells attached to the nanofilm surface only.

\subsection{Cell vitality, proliferation and morphology assessment}

Adhesion, proliferation and vitality of $\mathrm{C} 2 \mathrm{C} 12$ on PLA nanofilms were evaluated after 24,48 and $72 \mathrm{~h}$ in culture. The biocompatibility and the effect of the nanofilm on cells vitality were measured with two tests.

Cells were incubated at standard culture conditions on the nanofilm. The control sample is represented by cells cultured on Polystyrene (PS) standard culture wells. The number of viable cells was estimated with Trypan Blue assay (Sigma-Aldrich). The culture medium was removed from the Petri dish, thus allowing the adhesion of the nanofilm on the Petri dish surface. The cell monolayers onto the nanofilm surface were rinsed twice with PBS, detached from the substrate with trypsin, re-suspended with trypsin and DMEM, centrifuged at 2,000 rpm for $6 \mathrm{~min}$ and subsequently re-suspended in PBS. The detached cells were immediately stained with $0.4 \%$ Trypan Blue, and the number of viable cells was determined using a Burker chamber and light microscopy.

Vitality was further investigated using the LIVE/ DEAD ${ }^{\circledR}$ Viability/Cytotoxicity Kit (Molecular Probes, L3224). The kit contains Calcein AM (4 mM in anhydrous DMSO) and Ethidium homodimer-1 (EthD-1, $2 \mathrm{mM}$ in $\mathrm{DMSO} / \mathrm{H}_{2} \mathrm{O} 1: 4 \mathrm{v} / \mathrm{v}$ ), and it identifies live versus dead cells on the basis of membrane integrity and esterase activity. Cells were incubated for 24, 48 and $72 \mathrm{~h}$ at standard culture conditions, then the culture medium was removed and the cell monolayers onto the nanofilms surfaces were rinsed with PBS and treated for $10 \mathrm{~min}$ at $37^{\circ} \mathrm{C}$ with $2 \mu \mathrm{M}$ calcein $\mathrm{AM}$ and $4 \mu \mathrm{M}$ EthD-1 in PBS. Positive control was performed by incubating a sample with $50 \%$ DMSO for $15 \mathrm{~min}$. Cells were finally observed with an inverted fluorescent microscope (TE2000U, FITC-TRITC filters, Nikon) equipped with a cooled CCD camera (DS-5MC USB2, Nikon) and with NIS Elements imaging software.

In order to evaluate the spreading of the cells and to observe their early differentiation with the formation of myotubes, a second set of samples was cultivated for 3 days using standard culture medium and for additional 4 days with differentiating medium. A session of high resolution 

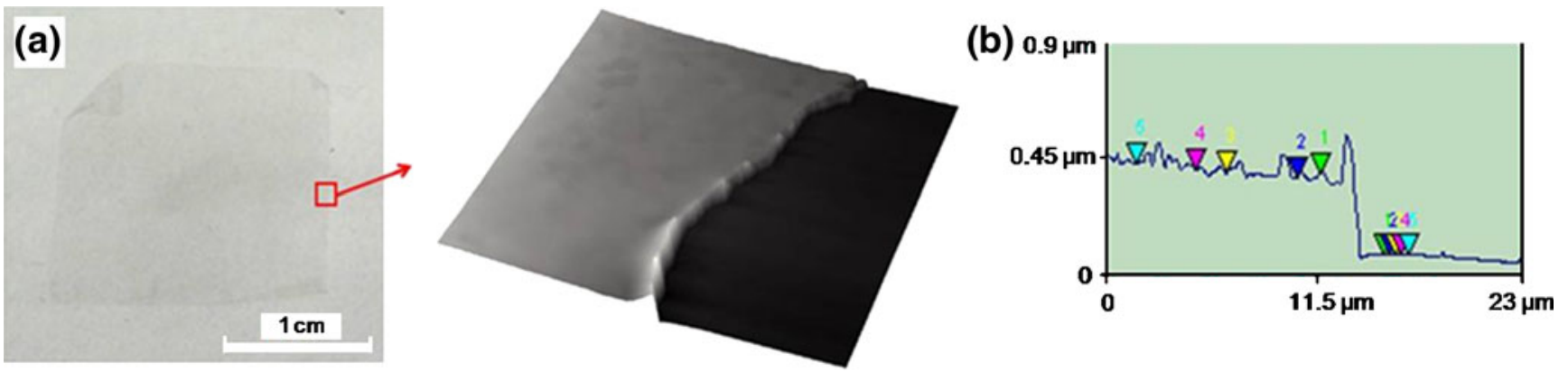

Fig. 3 (a) Optical and 3D AFM image of the nanofilm edge (height channel, scan window $100 \mu \mathrm{m} \times 100 \mu \mathrm{m}$ ); (b) Cross-sectional analysis of nanofilm edge thickness (scan window $23 \mu \mathrm{m} \times 23 \mu \mathrm{m}$ )

imaging was performed, analyzing cell shape and spreading onto the nanofilm surface with Atomic Force Microscopy (AFM) and Focused Ion Beam (FIB) microscopy.

For AFM analysis, the presence and the shape of elongated myotubes were evaluated 7 days after from cell seeding. The culture medium was removed, allowing the adhesion of the nanofilm on the bottom of the Petri dish. The nanofilm surfaces covered by cell monolayers were rinsed twice with deionized water and $10 \mu 1$ of $2 \%$ formalin solution were added. The samples were maintained at $4{ }^{\circ} \mathrm{C}$ for $15 \mathrm{~min}$, and afterwards on ice, with the Petri dish open in order to dry up, for $20 \mathrm{~min}$.

As regards FIB imaging, we chose a sample cultured for 3 days after cell seeding, in order to evaluate cell spreading and shape. Samples were prepared and fixed following the same procedure selected for AFM analysis and a final gold layer $(20 \mathrm{~nm})$ was deposed on the dried sample by chemical vapour deposition (RF Sputtering Sistec, model DCC 150) in order to have a conductive sample. The conductive coating enhances the contrast and brightness of the sample. In this work a FIB system FEI 200 (Focused Ion Beam for localized milling and deposition) was used, delivering a $30 \mathrm{keV}$ beam of gallium ions $\left(\mathrm{Ga}^{+}\right)$, with variable beam currents, ranging from $1 \mathrm{pA}$ up to $11 \mathrm{nA}$.

\section{Results}

\subsection{Nanofilm characterization}

The thickness of polymeric nanofilms depends on the selected spinning speed and time, and on the PLA concentration (Zhao and Marshall 2008). From AFM topographic imaging, it is possible to quantify the thickness of the nanofilm, measuring the height profile of the edge (Fig. 3(b)).

As shown in Fig. 3(b), the profile is quite homogeneous and the average thickness, calculated for 6 different samples, was around $320 \mathrm{~nm}$ obtained with the selected fabrication parameters (PLA 2 wt.\%, and 4,000 rpm for $20 \mathrm{~s}$ for spinner rotation). Lowering the scan range area from $100 \mu \mathrm{m} \times 100 \mu \mathrm{m}$ down to $10 \mu \mathrm{m} \times 10 \mu \mathrm{m}$, the topography of the polymeric film can be appreciated, and the roughness can be measured by software analysis. The average roughness of the nanofilm, as reported in Fig. 4, is measured as $\mathrm{R}_{\mathrm{A}}$. The profile curves oscillate around an average value of the roughness of $15 \mathrm{~nm} \pm 2 \mathrm{~nm}$.

The roughness value also can be correlated with the fabrication procedure, in particular with the polymer concentration and viscosity. The surface morphology of
Fig. 4 Software analysis of a topographical image used to measure surface roughness. For all tested samples, the average roughness RA was evaluated through the formation of specific line profiles
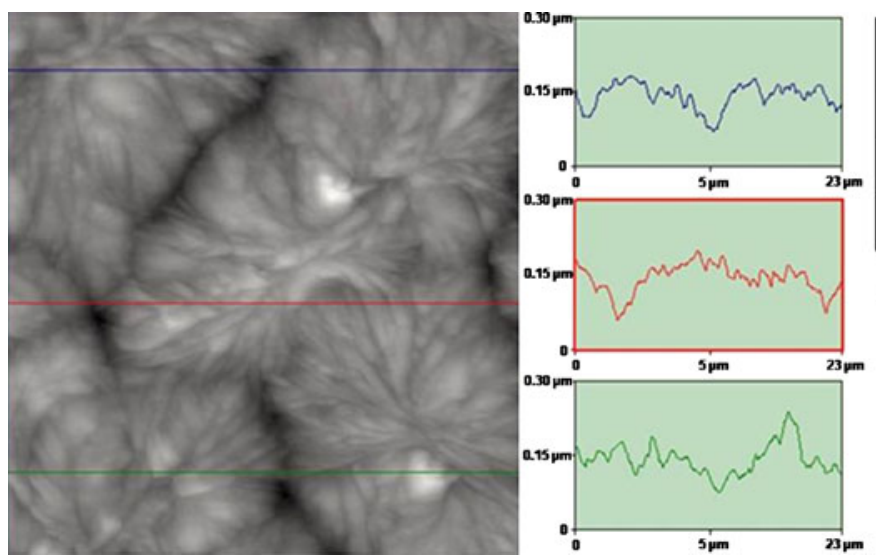

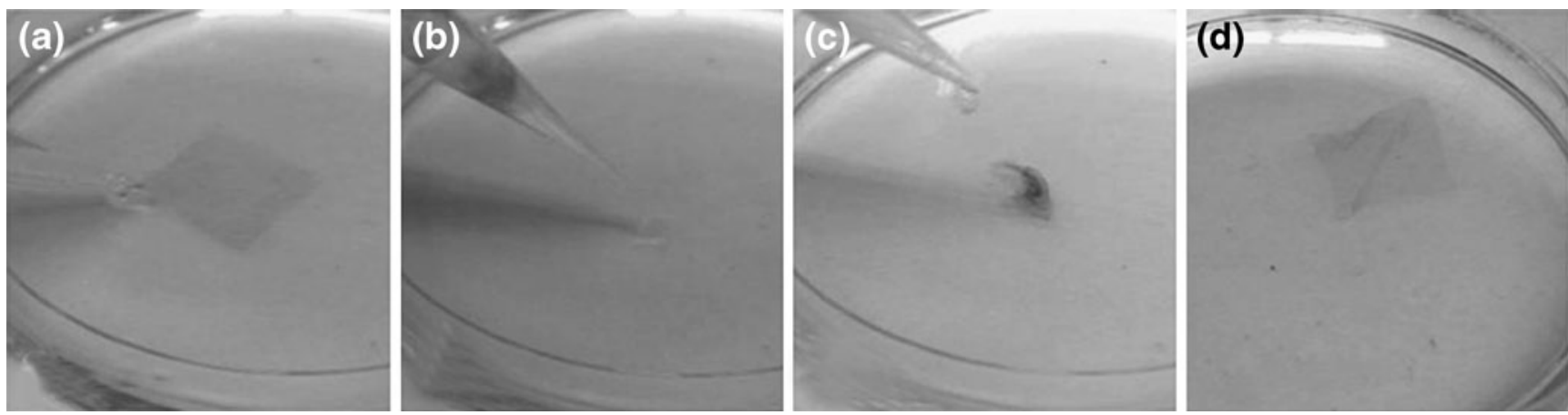

Fig. 5 Cyclic aspiration of a nanofilm in water: it can be easily aspirated $(\mathbf{a}, \mathbf{b})$ and ejected (c) through the micrometric hole of the pipette, without damaging the structure (d)

the PLA nanofilm in Fig. 4 showed remarkably crystalline structure of the assembled polymer.

The behavior of the single layer nanofilm is preliminarily evaluated in this work, by a visual assessment of the integrity of the film after repeated aspirations/injections cycles by using micropipettes (Fig. 5). From these tests, nanofilms result able to be aspired and injected for more than 10 cycles through a $0.75 \mathrm{~mm}$ hole fully maintaining their integrity. In order to investigate quantitative mechanical properties of the nanofilms we performed bulging test experiments on 6 different samples. We concluded that the

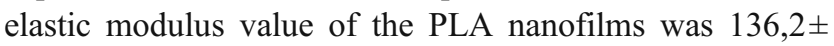
$43,5 \mathrm{MPa}$.
The possibility to manipulate the polymeric substrate, thanks to its flexibility, is a key issue for the use of this free-standing nanofilm as cell substrate. Moving, sterilizing and handling of the film in cell culture medium, by using traditional micropipettes, is thus possible without damaging the film structure.

\subsection{Cells viability and proliferation}

The results obtained by Trypan Blue exclusion, evaluated at 24, 48 and $72 \mathrm{~h}$ are summarized in Fig. 6.

As shown in Fig. 6, there is an evident proliferation of $\mathrm{C} 2 \mathrm{C} 12$ on the surface of the nanofilm. The comparison
Fig. 6 Cell counting and viability evaluated by Trypan Blue exclusion: (a) proliferation curve of the cells cultured on nanofilms compared with the control sample, observed after 24,48 and $72 \mathrm{~h}$ of incubation; (b) number of blue stained cells on nanofilms compared with the PS sample, after 24, 48 and $72 \mathrm{~h}$ of incubation; (c) density of cells cultured on nanofilms, compared with the number of cells cultured on PS substrate, observed after 24,48 and $72 \mathrm{~h}$ of incubation; (d) density of blue stained cells on nanofilms, compared with the number of blue stained cells on PS substrate, observed after 24, 48 and $72 \mathrm{~h}$ of incubation (a)

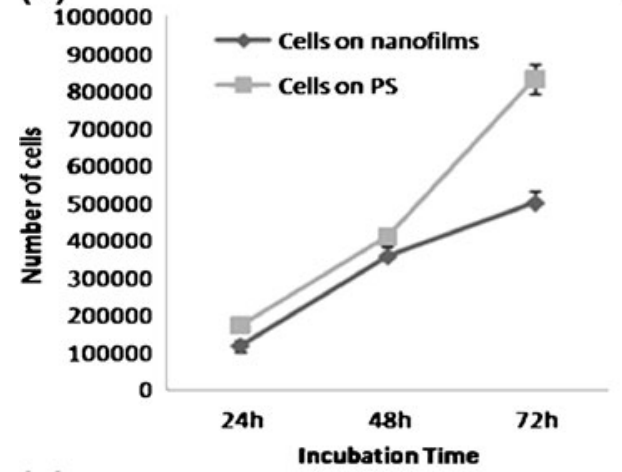

(c)

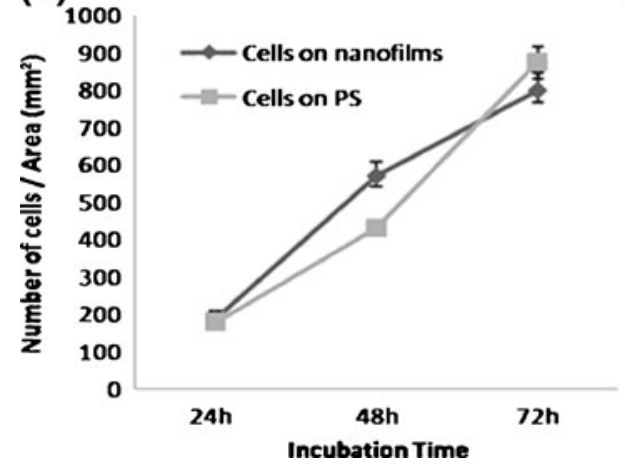

(b)

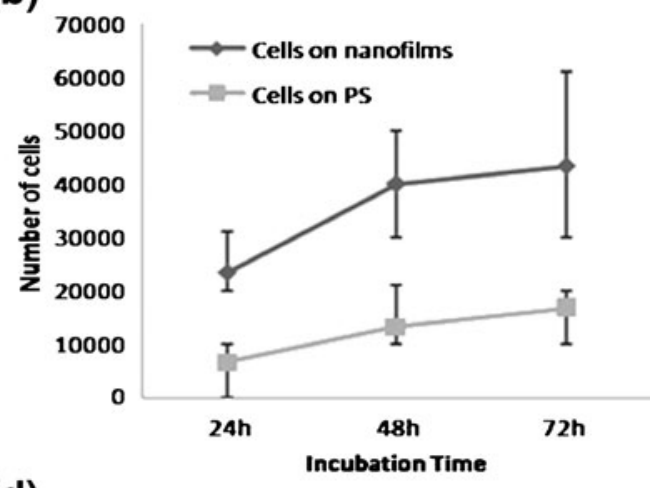

(d)

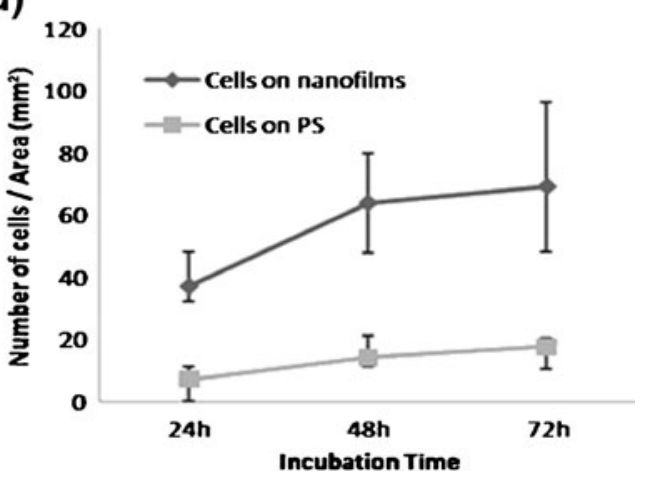


with the control values (Fig. 6(a)) reveals that cells adhere and proliferate less on PLA nanofilms respect to Polystyrene wells, but the difference is very small for what concerns samples incubated for 24 and $48 \mathrm{~h}$. This is due to the fact that the starting number of cells that adhere on the free-standing nanofilm is lower than the control sample because, after initial seeding, $65 \%$ of the total population of cells remains on the free-standing nanofilm, while the other part normally proliferates on the PS bottom. The cell density on the nanofilm is thus slightly limited in the following days. Figure 6(a) shows that the slopes of the proliferation curves for cells cultured on nanofilms and cells cultured on PS samples is the same, until $48 \mathrm{~h}$ from the seeding time. This leads to the conclusion that, until $48 \mathrm{~h}$ of culture, the duplication time of the cells ( $18 \mathrm{~h}$ in standard condition), is not significantly altered by the nanofilm. Furthermore, there is not any delay in the attachment and anchoring of the cells onto the freestanding nanofilm since the number of living cells increases gradually with the incubation time. For what concerns the samples incubated for $72 \mathrm{~h}$, the number of cells on Polystyrene wells is much higher than the number of cells on PLA nanofilms. This has not to be related to a lower proliferation rate induced by the PLA substrate, but to the different size of the samples. The nanofilm area is significantly smaller (about $625 \mathrm{~mm}^{2}$ ) respect to the control wells (about $950 \mathrm{~mm}^{2}$ ). A limited surface available for cells leads to an earlier approaching to the confluence, that reduces and finally stops cell proliferation. At $48 \mathrm{~h}$ of culture, the cells on the nanofilms start to approach confluence, and the slope of the proliferation curve between $48 \mathrm{~h}$ and $72 \mathrm{~h}$ of culture is lower. This is not true for the cells cultured on PS samples, that start to approach confluence at $72 \mathrm{~h}$. Between $48 \mathrm{~h}$ and $72 \mathrm{~h}$ of culture the slope of the proliferation curve increases, still fitting the first portion of the logistic curve for cell proliferation. However, if we compare the number of cells normalized by the sample area (Fig. 6(c)) we realize that there is no significant difference between nanofilms and PS samples. This confirms that PLA nanofilms
Fig. 7 Results of the vitality assay. Left images are bright field images of the samples incubated for 24 (a), 48 (c) and $72 \mathrm{~h}$ (e). Right images are the corresponding fluorescent images at 24 (b), 48 (d) and $72 \mathrm{~h}$ (f). Scale bars correspond to $100 \mu \mathrm{m}$
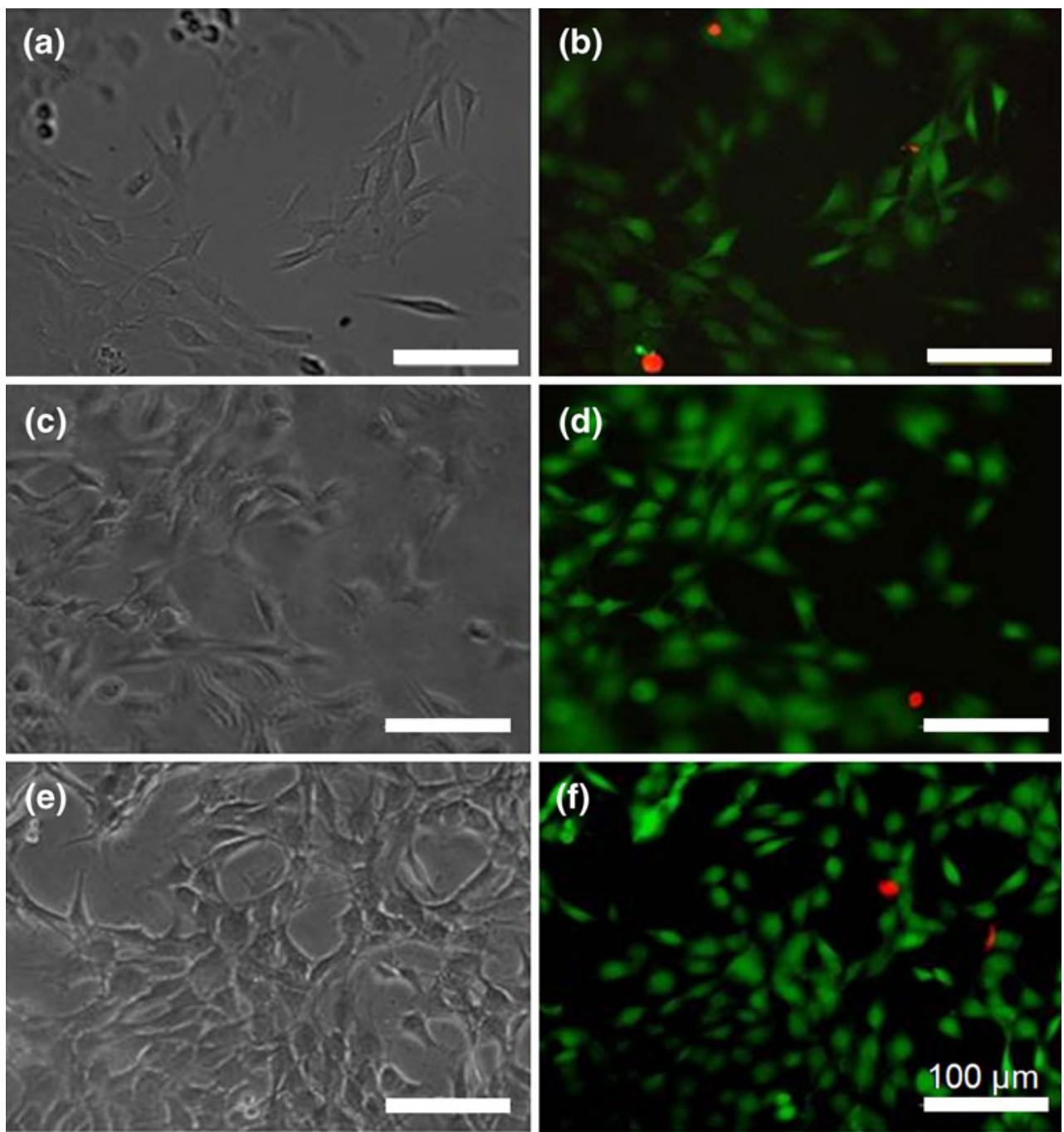
represent a good surface for $\mathrm{C} 2 \mathrm{C} 12$ anchoring, spreading and proliferation.

This is also confirmed by the results shown in Fig. 6(b) and (d). Dead and apoptotic cells number does not increase in a dramatic way on the nanofilm samples with the incubation time, and it is comparable with the results on Polystyrene samples. The mortality of cells on nanofilms is higher, but this can be due to the stresses that cells feel during the initial operations of manipulation and seeding of the nanofilms.

Figure 7 summarizes results obtained with LIVE/DEAD ${ }^{\circledR}$ assay. The green stained cells are live cells attached to the nanofilms, while the red colored cells are the apoptotic ones. The limited number of dead and apoptotic cells (which reaches a percentage of $7 \%$ at $72 \mathrm{~h}$ ) confirms the vitality of cells attached on the nanofilm surface.

\subsection{Cells attachment and spreading}

In order to better investigate cell spreading on the floating nanofilm surface, two samples were analyzed with high resolution microscopy techniques. Figure 8 shows an image of a PLA nanofilm with adherent cells after $24 \mathrm{~h}$ in culture, obtained by FIB. The nanofilm is partially covered by a monolayer of myoblasts, with rounded nuclei that are clearly visible (red arrows). The population appears as a carpet of cells, with a homogeneous adhesion and spreading because of the secretion of adhesive proteins that cover the entire nanofilm. This image confirms that there is a good adhesion of $\mathrm{C} 2 \mathrm{C} 12$ on the flexible substrate as observed from the proliferation assay.

The adhesion of the myoblasts is governed by the formation of a highly branched network of proteins and extracellular matrix components, and for this reason the spreading and focal adhesion of single cells cannot be distinguished clearly. Figure 7 shows that before the differentiation phase, cells adhere and proliferate in growth medium and that the PLA nanofilm promoted the formation of focal adhesions and organization of the cytoskeleton, as well as proliferation. This is an important parameter for evaluating the biocompatibility of the PLA nanofilm, suggesting an initial and good mechanical matching between the two parts. However, after 4 days with low serum differentiating medium, the dimension and the thickness of the cells change significantly. They abandon the round shape, thus assuming an elongated aspect. Figures 8 and 9 report the topographical profiles obtained by AFM of a single cell and a single myoblast, respectively, observed on single samples. In Fig. 8 the cell is fixed after $48 \mathrm{~h}$ of incubation in standard serum medium (10\% FBS). Measuring the height profile at the cell nucleus level, an average value of $0,97 \pm 0,3 \mu \mathrm{m}$ is obtained, suggesting that the cell is assuming an almost flat appearance. The thickness of the cell, which can be

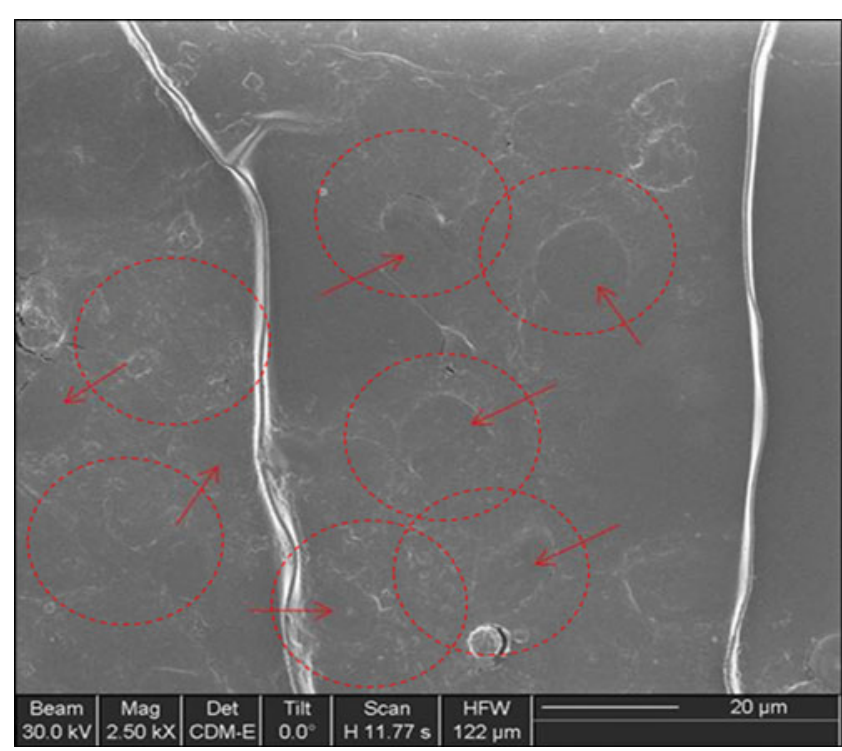

Fig. 8 FIB image of cells spread onto the nanofilm surface; sample incubated for $24 \mathrm{~h}$

correlated with the maximum peak-to-valley height $R_{t}$, reaches a maximum level of $1 \pm 0.2 \mu \mathrm{m}$, confirming that the complete cell body adheres on the nanofilm, whose thickness is negligible.

The middle term biocompatibility of nanofilms and their capability to allow $\mathrm{C} 2 \mathrm{C} 12$ differentiation into myotubes was also evaluated, substituting the culture medium with a low serum medium (1\% FBS). Figure 9(a) shows cells on nanofilm cultured for 7 days with standard serum medium (10\% FBS). The cells reach the confluence, maintaining a compliant adhesion to the nanofilm. This evidences that the anchoring of the cells is maintained at 7 days in culture on the flexible substrate without losing contact between myoblasts and nanofilm.

A reorganization is needed for cell striation, which leads to an early differentiation stage, with the fusion of cells into firmly adherent myotubes. Figures 9 (b) and 10 shows a bright field image obtained by AFM of $\mathrm{C} 2 \mathrm{C} 12$ (1 week of culture on the nanofilm surface). This sample was cultured for 3 days with standard serum medium (10\% FBS) and for 4 more days with low serum medium (1\% FBS), allowing the formation of myotubes.

\section{Discussion and conclusions}

Since free-standing flexible ultra-thin films show a nanometric and controllable thickness, good manipulation in liquid, and they can show a high elastic modulus (the elastic modulus of our nanofilms is around $136 \mathrm{MPa}$, that is 
Fig. 9 AFM image of a single cell spreading onto the nanofilm surface. Sample incubated for 48 h. (a) Three-dimensional representation of the cell onto the nanofilm (scan area); (b) software analysis for the measurement of the cell thickness respect to the PLA surface plane

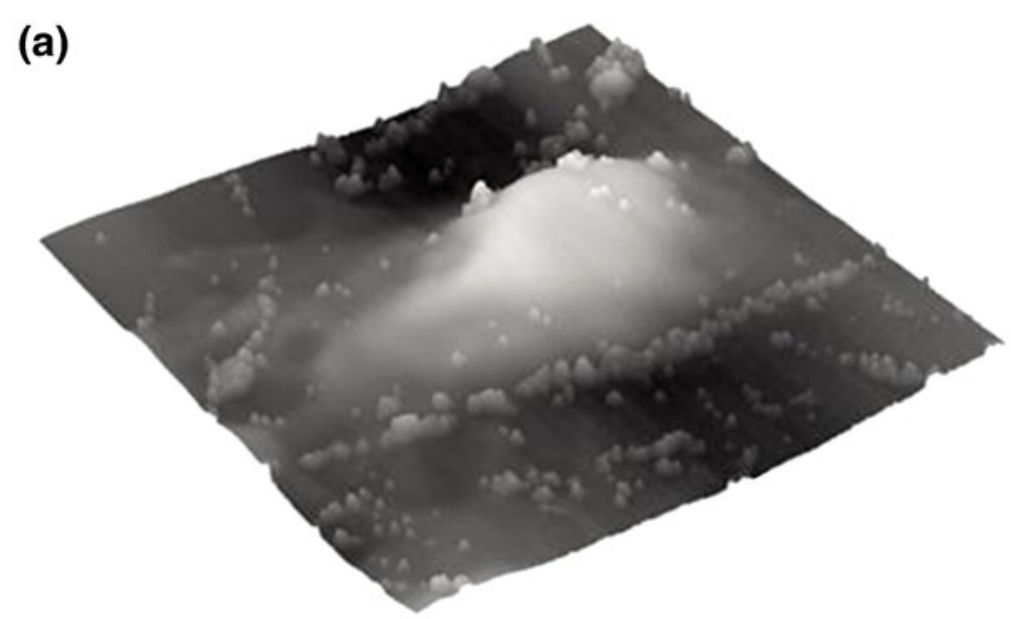

(b)

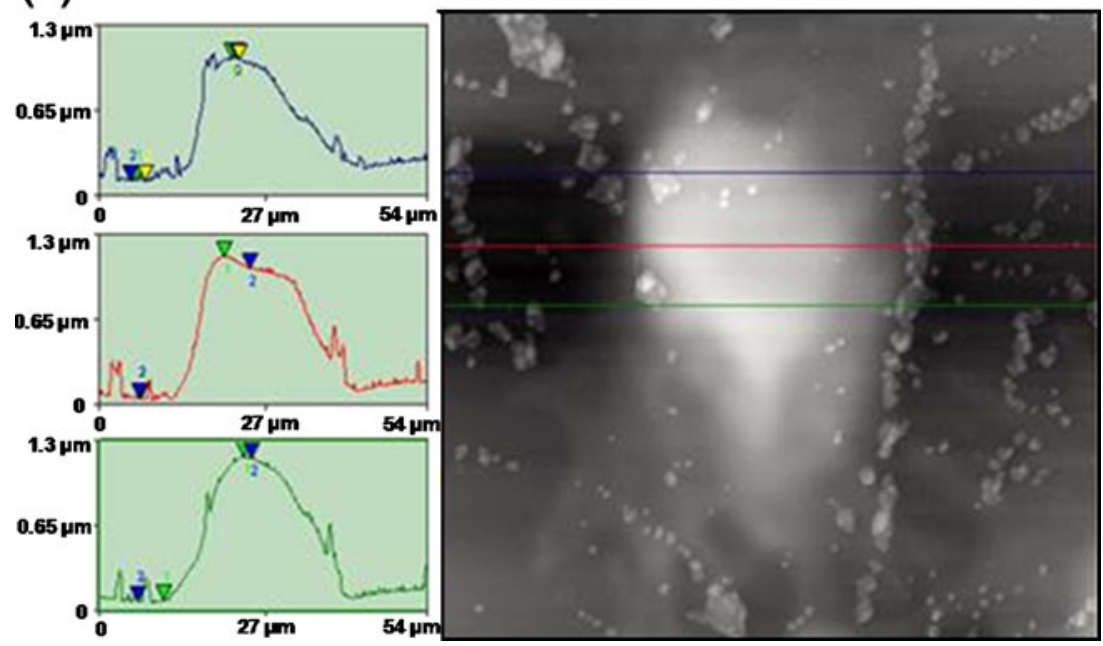

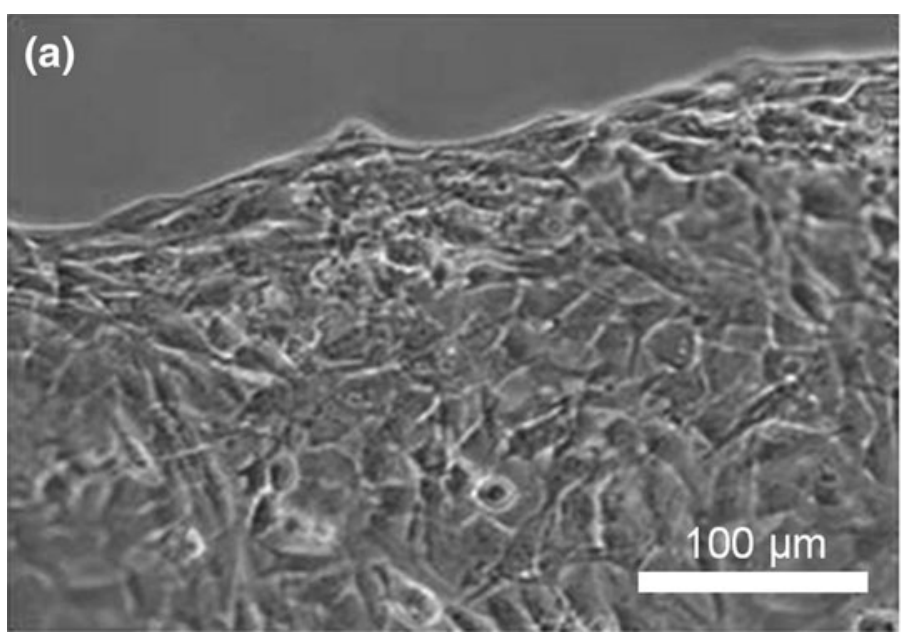

(b)

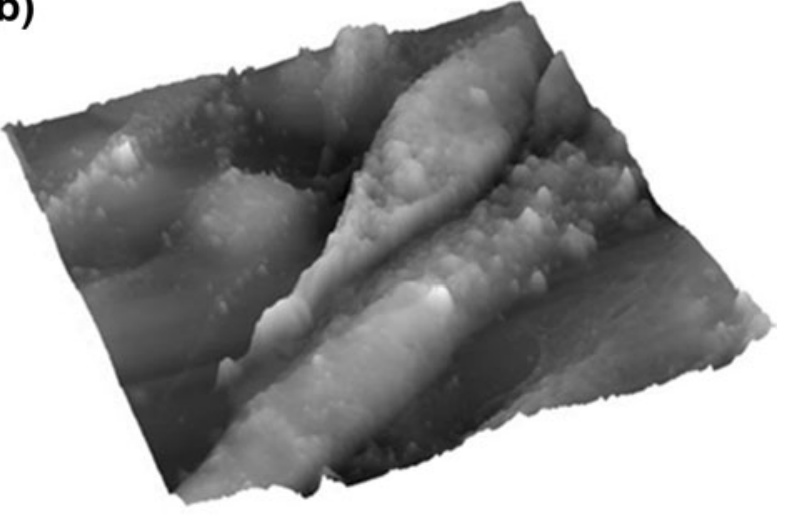

Fig. $10 \mathrm{C} 2 \mathrm{C} 12$ cells after 1 week in culture on PLA nanofilms: (a) bright field image of a free-standing in liquid nanofilm, covered by skeletal muscle cells cultured for 1 week with standard serum medium (10\% FBS); (b) AFM image of elongated early differentiated myoblasts on the PLA surface, cultured for 3 days with standard serum medium (10\% FBS) and 4 days with low serum medium $(1 \%$ FBS) 
a good value for $\mathrm{C} 2 \mathrm{C} 12$ culture and differentiation), we envisage the possibility to use them as biocompatible artificial components for the design of muscle constructs in vitro and for the development of new bio-hybrid devices. Based on this premise, in this work skeletal muscle cells were chosen and viability, adhesion, proliferation and early differentiation were monitored for 1 week on single layer poly(lactic acid) nanofilm with thickness of $\sim 300 \mathrm{~nm}$, which is two order of magnitude lower compared to PDMS thin films already used as flexible support for bio-hybrid devices (Tanaka et al. 2007b (II)).

Preliminary results about short term biocompatibility (until $72 \mathrm{~h}$ ) of PLA nanofilms are reported, as key issue for the use of these substrates for cell culture. Generally, the free-standing nanofilms show a homogeneous cell adhesion over their surface, without significant alteration of the duplication time and proliferation. This suggests that the fluidodynamic stress on the free-standing nanofilm does not interfere with cell adhesion and growth. Moreover, the film properties, in terms of roughness, thickness and flexibility do not influence the spreading of the cells and the organization of a network of adhesive proteins in the first days. When the differentiation starts, the reorganization of the cells and the striation phase are allowed, thus fostering the possibility to reach the myotubes formation and ultimately their contraction. Studies in literature (Ren et al. 2008) reveal that stiff films $\left(\mathrm{E}_{0}>320 \mathrm{kPa}\right)$ promoted formation of focal adhesions and organization as well as differentiation into myotubes. The elastic modulus of our PLA nanofilms is higher than this reference value, but it is much smaller respect to the common values highlighted in literature (Okamura et al. 2009). This, together with the particular crystalline structure and roughness, reflects in a good cell adhesion and proliferation on the nanofilm surface, still not shown in literature for PLA ultra-thin films. In order to better quantify the focal adhesions, the spreading and the different phases of cell differentiation, future tests will be performed, such as staining actin, myosin and vinculin with fluorescent tags before and after the use of low serum differentiating medium. Evaluation of myotubes formation and striation will be also performed, considering quantitative parameters as the fusion index, the aspect ratio and the surface areas of the myotubes (Ren et al. 2008). In this framework, the nanometric thickness of PLA nanofilms could permit an almost "mechanically substrate-independent" contraction of muscle cells cultured and differentiated on their surface. The attached myotubes should easily contract the flexible nanofilm, thus inducing the substrate displacement or its shortening and elongation.

Given the above properties, these nanofilms appear as appropriate substrates for realizing self-contracting muscle films with cardiomyocytes, as reported by [Personal communication by Prof. Keisuke Morishima]. First results on patterning adhesion proteins for guiding cell growth and myotubes organization have been already recorded.

Acknowledgements The authors would like to thank the Italian Ministry of Foreign Affairs, General Directorate for Cultural Promotion and Cooperation for supporting the collaboration between the authors' group. In addition, they wish to thank Mr. Carlo Filippeschi for technical support during the clean-room activities.

\section{References}

A.D. Bach, J.P. Beier, J. Stern-Staeter, R.E. Horch, Skeletal muscle tissue engineering. J. Cell. Mol. Med. 10(3), 413-422 (2004)

H.M. Blau, C.P. Chiu, C. Webster, Cytoplasmic activation of human nuclear genes in stable heterocaryons. Cell 32(4), 1171-1180 (1983)

D.E. Discher, P. Janmey, Y. Wang, Tissue cells feel and respond to the stiffness of their substrate. Science 310(5751), 1139-1143 (2005)

A.J. Engler, M.A. Griffin, S. Sen, C.G. Bönnemann, H.L. Sweeney, D. E. Discher, Myotubes differentiate optimally on substrates with tissue-like stiffness pathological implications for soft or stiff microenvironments. J. Cell Biol. 166(6), 877-887 (2004)

A.W. Feinberg, A. Feigel, S.S. Shevkoplyas, S. Sheehy, G.M. Whitesides, K.K. Parker, Muscular thin films for building actuators and powering devices. Science 317(5843), 1366-1370 (2007)

T. Fujie, Y. Okamura, S. Takeoka, Ubiquitous transference of a freestanding polysaccharide nanosheet with the development of a nano-adhesive plaster. Adv. Mater. 19, 3549-3553 (2007)

H. Fujita, T. Nedachi, M. Kanzaki, Accelerated de novo sarcomere assembly by electric pulse stimulation in C2C12 myotubes. Exp. Cell Res. 313(9), 1853-1865 (2007)

H. Herr, R.G. Dennis, A swimming robot actuated by living muscle tissue. J. Neuroeng. Rehabil. 1, 6 (2004)

Y. Hiratsuka, M. Miyata, T. Tada, T.Q.P. Uyeda, A microrotary motor powered by bacteria. Proc. Natl Acad. Sci. 103, 13618-13623 (2006)

C.K. Huang, W.M. Lou, C.J. Tsai, T.C. Wu, H.Y. Lin, Mechanical properties of polymer thin film measured by the bulge test. Thin Solid Films 515(18), 7222-7226 (2007)

J.A. Hubbell, Bioactive biomaterials. Curr. Opin. Biotechnol. 10(2), 123-129 (1999)

T. Ishibashi, Y. Hoshino, H. Kaji, M. Kanzaki, M. Sato, M. Nishizawa, Localized electrical stimulation to $\mathrm{C} 2 \mathrm{C} 12$ myotubes cultured on a porous membrane-based substrate. Biomed. Microdevices 11(2), 413-419 (2008)

D. Kim, J. Park, K.Y. Suh, P. Kim, S.K. Choi, S. Ryu, S. Park, A.H. Lee, B. Kim, Fabrication of patterned micromuscles with high activity for powering biohybrid microdevices. Sens. Actuators B 117, 391-400 (2006)

K. Morishima, Y. Tanaka, M. Ebara, T. Shimizu, A. Kikuchi, M. Yamato, T. Okano, T. Kitamori, Demonstration of a biomicroactuator powered by cultured cardiomyocytes coupled to hydrogel micropillars. Sens. Actuators B 119, 345-350 (2006)

Y. Okamura, K. Kabata, M. Kinoshita, D. Saitoh, S. Takeoka, Freestanding biodegradable poly(lactic acid) nanosheet for sealing operations in surgery. Adv. Mater. 21, 1-5 (2009)

V. Pensabene, V. Mattoli, T. Fujie, A. Menciassi, S. Takeoka, P. Dario, Magnetic nanosheet adhesion to mucosal tissue, in Proc. IEEE Nano, (Genova, 2009), pp. 489-493

V. Raffa, V. Pensabene, A. Menciassi, P. Dario, Design criteria of neuron/electrode interface. The focused ion beam technology as an analytical method to investigate the effect of electrode surface 
morphology on neurocompatibility. Biomed. Microdevices 9(3), 371-383 (2007)

K. Ren, T. Crouzier, C. Roy, C. Picart, Polyelectrolyte multilayer films of controlled stiffness modulate myoblast cells differentiation. Adv. Funct. Mater. 18(9), 1378-1389 (2008)

A. Sathaye, N. Bursac, S. Sheehy, L. Tung, Electrical pacing counteracts instrinsic shortening of action potential duration of neonatal rat ventricular cells in culture. J. Mol. Cell. Cardiol. 41, 633-641 (2006)

H. Shin, S. Jo, A.G. Mikos, Review: biomimetic materials for tissue engineering. Biomaterials 24, 4352-4364 (2003)

Y. Tanaka, K. Morishima, T. Shimizu, A. Kikuchi, M. Yamato, T. Okano, T. Kitamori, Demonstration of a PDMS-based biomicroactuator using cultured cardiomyocytes to drive polymer micropillars. Lab Chip 6, 230-235 (2006)

Y. Tanaka, K. Sato, T. Shimizu, M. Yamato, T. Okano, T. Kitamori, Biological cells mon microchips: new technologies and applications. Biosens. Bioelectron. 23, 449-458 (2007a)

Y. Tanaka, K. Sato, T. Shimizu, M. Yamato, T. Okano, T. Kitamori, A micro-spherical heart pump powered by cultured cardiomyocytes. Lab Chip 7(2), 207-212 (2007b)

Z. Tang, Y. Wang, P. Podsiadlo, N.A. Kotov, Biomedical applications of Layer-by-Layer assembly: from biomimetics to tissue engineering. Adv. Mater. 18(24), 3203-3224 (2006)

H. Vandenburgh, J. Shansky, F. Benesch-Lee, V. Barbata, J. Reid, L. Thorrez, R. Valentini, G. Crawford, Drug-screening platform based on the contractility of tissue-engineered muscle. Muscle Nerve 37, 438-447 (2008)

R. Vendamme, S.Y. Onoue, A. Nakao, T. Kunitake, Robust freestanding nanomembranes of organic/inorganic interpenetrating networks. Nat. Mater. 5(6), 494-501 (2006)

N.R. Washburn, K.M. Yamada, C.G. Simon, S.B. Kennedy, E.J. Amis, High-throughput investigation of osteoblast response to polymer crystallinity: influence of nanometer-scale roughness on proliferation. Biomaterials 25(7-8), 1215-1224 (2004)

D.B. Weibel, P. Garstecki, D. Ryan, W.R. DiLuzio, M. Mayer, J.E. Seto, G.M. Whitesides, Microoxen: microorganisms to move microscale loads. Proc. Natl. Acad. Sci. 102, 11963-11967 (2005)

K. Wilson, P. Molnar, J. Hickman, Integration of functional myotubes with a Bio-MEMS device for non-invasive interrogation. Lab Chip 7, 920-922 (2007)

J. Xi, J.J. Schmidt, C.D. Montemagno, Self-assembled microdevices driven by muscle. Nat. Mater. 4, 180-184 (2005)

D. Yaffe, O. Saxel, A myogenic cell line with altered serum requirements for differentiation. Differentiation 7(3), 159-166 (1977)

K. Yamasaki, H. Hayashi, K. Nishiyama, H. Kobayashi, S. Uto, H. Kondo, S. Hashimoto, T. Fujisato, Control of myotube contraction using electrical pulse stimulation for bio-actuator. J. Artif. Organs 12(2), 131-137 (2009)

Y. Zhao, J.S. Marshall, Spin coating of a colloidal suspension. Phys. Fluids 20(4), 043302 (2008) 